\title{
Preliminary Study for Construction of the Developmental Disability Corpus: Focus on Speech-Language Pathologists Survey Responses
}

\author{
Whasoo Kim', Sangjun Lee ${ }^{2}$ \\ 'Department of Speech-Language Pathology, Daegu University, Gyeongsan, Korea \\ ${ }^{2}$ Major in Speech-Language Pathology, Department of Rehabilitation Science, Graduate School, Daegu University, Gyeongsan, Korea
}

\section{발달장애인 말뭉치 구축을 위한 예비연구: 언어재활사의 설문 반응을 중심으로}

김 화 수 ${ }^{1}$ 이 상 준 ${ }^{2}$

대구대학교 언어치료학과', 대구대학교 일반대학원 재활과학과 언어치료전공 ${ }^{2}$

\begin{abstract}
Purpose: This study is a preliminary study for analyzing the written and spoken language of developmental disabilities and constructing the developmental disability corpus. Methods: This study surveyed 174 persons consisting of speech-language pathologists (SLPs), researchers and college students. This survey was conducted on the internet, and designed to utilization experience of the corpus, representative written and/or spoken language, and additional information. Results: First, the perception and utilization experience of the corpus were very low. Second, the SLPs response to the representative written language of developmental disabilities was 'text in class' and spoken language was 'conversation with family and other people'. Third, most of the participants needed 'Language Level' in personal information and 'conversation situation' in additional information. Conclusion: The results of this study will be used as preliminary data for construction of the developmental disorders corpus.
\end{abstract}

Key Words: Developmental disabilities, Corpus, Speech-Language Pathologist, Communication.

Received: June 5, 2017 / Revised: June 25, 2017 / Accepted: June 26, 2017

Correspondence: Sangjun Lee, Major in Speech-Language Pathology, Department of Rehabilitation Science, Graduate School, Daegu University, 201 Daegudae-ro, Jillyang-eup, Gyeongsan 38453, Korea

Tel: +82-53-850-4324 / Fax: +82-53-850-4329 / E-mail: a376100@naver.com

\section{INTRODUCTION}

발달장애인이란 장애인복지법 제2조1항에 따르면 장애인으 로 지적장애인과 자폐성장애인 및 그 밖에 통상적인 발달이 나 타나지 아니하거나 크게 지연되어 있는 사람을 의미한다. 발달 장애인은 다른 장애유형과 달리 자립 역량이 부족해 타인의 보 호를 받아야 한다(Kwon, 2016). Kim et al.(2014)의 조사 결과 에 따르면 발달장애인은 다른 장애인에 비해 집밖 활동이 불편 하다는 의견이 상대적으로 높았다. 특히, 자폐성장애인의 $81.6 \%$ 가 불편하다고 느끼며 불편 정도가 매우 컸는데, 이는 동반자의 부재나 의사소통의 어려움 때문이라는 것이다.
이러한 사회적 문제를 해소하기 위하여 2014년 4월 29일 국 회를 통과한 “발달장애인 권리보장 및 지원에 관한 법률”(약칭 '발달장애인법')은 같은 해 5월 20일(법률 제12618호)에 고시되 었다(Kwon, 2016). 발달장애인법은 발달장애인의 생애주기에 따른 특성 및 복지 욕구에 적합한 지원 등을 효율적으로 제공 하여 사회참여 촉진, 권리 보호 및 인간다운 삶을 살 수 있는 것을 목적으로 한다. 이 법은 발달장애인이 살아가며 경험하는 어려움에 대해 사회적인 공감과 인정을 이끌어 내며 지원에 대 한 제도적 근거를 마련하였다는 점에서 큰 의미를 가진다. 발달 장애인법의 제 10 조 의사소통지원에 따르면, 국가와 지방단체는 발달장애인의 권리와 의무에 중요한 정책정보를 발달장애인이 
이해하기 쉬운 형태로 작성 및 배포하여야 하며, 발달장애인이 자신의 의사를 원활하게 표현할 수 있도록 학습에 필요한 의사 소통 도구 개발, 교육 및 전문 인력을 양성하며, 민원담당 직원 이 발달장애인과 효과적으로 의사소통할 수 있는 지침을 개발 및 교육을 실시하여야 한다. 각 항목에 필요한 세부사항들의 경 우 대통령령을 통해서 지정하고 있지만, 아직 국가 차원의 발달 장애인법 시행에 따른 구체적인 정책정보 작성 기준, 의사소통 지침의 개발 및 교육 기준, 전문 인력 양성에 따른 계획은 정해 지지 않은 상태이다. 특히 중요한 정책 자료인 선거 안내물 등은 발달장애인을 위해 맞춤형으로 제작되지만, Kim et al.(2016)의 연구결과에 따르면 중증 발달장애인들은 이러한 자료에 대한 이해에도 어려움을 겪었다. 이 연구에서는 대안으로 중증 발달 장애인이 이해하기 쉬운 선거 관련 정보를 제공하는 것이 필요 하다고 하였으며, 발달장애인을 위한 개별 투표용지 및 시범 동 영상의 필요성에 대해서 강조하였다. 이번 제19대 대통령선거에 서 반응형 웹 콘텐츠인 '투표 미리하기 앱'과 애니메이션을 제작 하였으나, 발달장애인의 실제 언어사용 양상이나 관련 전문가 의 의견이 참고되었는지는 알 수 없다.

최근 컴퓨터와 소프트웨어의 발달로 인하여 빅데이터에 관 한 관심이 급속히 늘어가고 있다. 이는 정보통신기술의 발달 및 정보의 효율적인 저장, 분석이 가능하게 하며, 이를 이용하여 의 료 및 마케팅, 생물 정보학 등 많은 분야에서 빅데이터가 사용되 고 있다(Kim \& Cho, 2013). 이러한 연구가 언어학에서도 이루 어지고 있는데, 그것은 바로 '말뭉치 언어학(corpus linguistics)' 이다. 말뭉치란 실제 사람들에게 사용되는 언어의 모습을 보여 줄 수 있는 자료의 집합으로서, 대표성과 균형성, 대규모성이라 는 특성을 지닌다(Seo \& Han, 1999). 이러한 말뭉치 언어학은 언어 연구에 실증적인 자료를 제공하며 응용언어학 연구에도 발전을 가져왔다(Nam, 2014). Choe(2014)는 말뭉치 언어학이 많이 논의되는 이유 중 하나가 바로 말뭉치 언어학이 추구하는 방식에 있다고 하였다. 말뭉치 자료를 이용한 연구는 객관적 방 식을 활용한 대규모 언어자원을 바탕으로, 재현 가능한 언어적 일반화를 구축하여 추출 방식에 따라 다양한 형태로 정보를 가공할 수 있어 여러 응용언어학에서도 많이 사용된다.

그동안 이루어진 발달장애인의 언어와 관련된 대부분의 연 구들은 표준화된 검사를 바탕으로 동일 언어연령이나 생활연령 의 사람들과 비교하는 연구이며, 자발화를 대규모로 분석하는 연구는 거의 없었다(Kim et al., 2017). 자발화와 관련된 연구라 도 대부분의 연구는 자연스러운 발화보다는 연구자가 제시하 는 특정 상황이나 그림, 애니메이션 등과 관련된 발화를 분석하 는 연구였다. 발달장애인의 실제 언어 양상 및 차이점 등에 대 한 상세한 분석이 필요하며, 이를 위해서는 최근에 발전된 컴퓨 터공학 및 통계학을 활용하여 말뭉치 언어학을 사용할 수 있
다. 따라서 본 연구에서는 발달장애인의 문어와 구어를 분석하 여 이를 말뭉치로 구축하기에 앞서 발달장애인의 언어적 특성 에 대해 잘 알고 있는 전문가 집단인 언어재활사 및 연구자, 관 련 전공의 대학생들을 대상으로 말뭉치에 대한 지식 및 요구도 와 대표성이 있는 자료 구축에 관한 설문조사를 실시하였다. 이러한 설문을 통해 발달장애인 언어 자료 수집과 말뭉치 구축 시 고려해야 할 사항들을 조사하고, 본 연구의 결과를 실제 구 축 방향 설정에 참고하고자 하였다.

\section{MATERIALS AND METHODS}

\section{연구 대상}

본 연구는 2016년 4월 한 달 동안 발달장애인의 언어적 특성 에 대해 잘 알고 있는 전문가 집단인 언어재활사 및 연구자, 관 련 전공의 대학생 174 명을 대상으로 설문조사를 실시하였다. 연구 대상자의 일반적인 정보 항목은 대상자의 성별, 나이, 최 종학력, 소속기관, 자격급수, 치료 경력, 언어치료 관련 영역에 따라 7개의 항목으로 분류하였다. 대상자의 일반적 배경정보는 Table 1과 같다.

Table 1. Participants' general information

\begin{tabular}{|c|c|c|c|}
\hline Category & Type & $\mathrm{n}$ & $\%$ \\
\hline \multirow[t]{2}{*}{ Sex } & Female & 155 & 89.1 \\
\hline & Male & 19 & 10.9 \\
\hline \multirow[t]{3}{*}{ Age } & $20-29$ & 142 & 81.6 \\
\hline & $30-39$ & 25 & 14.4 \\
\hline & Over 40 & 7 & 4.0 \\
\hline \multirow[t]{6}{*}{ Workplace } & University & 67 & 38.5 \\
\hline & Speech clinic & 74 & 42.5 \\
\hline & Welfare center & 15 & 8.6 \\
\hline & Hospital & 8 & 4.6 \\
\hline & $\begin{array}{l}\text { Education office and } \\
\text { special-education school }\end{array}$ & 4 & 2.3 \\
\hline & Nursery & 6 & 3.4 \\
\hline \multirow[t]{3}{*}{ Certification steps } & Level 1 & 6 & 3.4 \\
\hline & Level 2 & 123 & 70.7 \\
\hline & Acquisition schedule & 45 & 25.9 \\
\hline \multirow[t]{5}{*}{ Clinical experience } & Under 1 year & 107 & 61.5 \\
\hline & $1-3$ years & 38 & 21.8 \\
\hline & $3-5$ years & 10 & 5.7 \\
\hline & $5-10$ years & 15 & 8.6 \\
\hline & Over 10 years & 4 & 2.3 \\
\hline \multirow[t]{4}{*}{ Position } & $\begin{array}{l}\text { Speech-language patholgy } \\
\text { major college students }\end{array}$ & 58 & 33.3 \\
\hline & SLP & 63 & 36.2 \\
\hline & Researcher & 22 & 12.6 \\
\hline & SLP and researcher & 31 & 17.8 \\
\hline
\end{tabular}

SLP: speech-language pathologist 


\section{자료 수집 절차}

본 연구는 언어재활사 및 연구자, 관련 전공의 대학생에게 연 구의 목적 및 말뭉치에 대한 정보를 제공하고 설문조사를 실시 하였다. 설문 응답은 자기 보고 형식으로, 스스로 일반적 정보 와 말뭉치의 활용여부 및 목적, 구축에 관한 의견에 대하여 응 답하도록 하였다. 자료 수집은 구글 설문지를 활용하여 모바일 및 인터넷으로 응답할 수 있도록 하였고, 총 174 명의 응답 결과 를 분석에 적용하였다.

\section{설문지 구성 및 자료 분석}

본 연구의 설문지는 Kang et al.(2015), Kwak et al.(2015), Seo et al.(2010)이 한국어 학습자 말뭉치 구축을 위하여 해당 분야의 전문가들을 상대로 진행했던 설문 문항을 본 연구의 목적에 맞게 수정 및 보완하였다. 설문지에 사용된 용어 및 구 성이 타당한지 검증하기 위하여 언어치료 전공 박사과정 5 인과 언어치료 전공의 교수 1 인의 감수를 받았으며, 발달장애인 치 료 경험이 있는 2 급 언어재활사 10 인을 대상으로 예비조사를 실시하고 보완 및 재구성하여 활용하였다.

설문지의 구성은 말뭉치 활용 여부 및 목적, 말뭉치 구축에 관한 의견으로 이루어졌다. 말뭉치 활용 여부 및 목적에는 말뭉 치에 대한 사전정보 유무 및 활용 경험, 향후 활용 의사 및 목 적, 내용의 5 개의 항목으로 분류하였고, 활용 경험 유무에 따라 4 개의 항목을 추가로 물었다. 말뭉치 구축에 관한 의견은 문어 적 특징, 구어적 특징, 추가되는 개인정보, 개인정보 외의 환경/ 상황/참여자정보 여부에 따라 4가지 항목으로 구성되었으며, 환경과 상황, 참여자정보에 대해 추가적으로 얻어야 할 정보 및 고려해야 할 사항에 대한 3가지 문항이 추가되었다.

각 하위 문항들은 명목척도에 따라 응답하도록 하였으며, 기 타 의견이 있다면 자신의 의견을 작성하여 제출하도록 하였다.

\section{RESULTS}

\section{말뭉치에 대한 지식 및 요구도}

\section{말뭉치에 대한 인식도 및 활용 경험}

Table 2를 보면 전체 응답자 174명 중 133명(76.4\%)은 말뭉치 (corpus)라는 용어를 들어본 적이 없었으며, 활용 경험이 있는 응답자는 8명(4.6\%)에 불과하였다.

\section{말뭉치의 활용 목적 및 내용, 습득 경로}

Table 3에 나타난 바와 같이 말뭉치를 활용해 본 경험이 있 는 응답자 8 명의 의견을 중복 가능하게 하여 수집하였으며, 응 답자의 6명(75.0\%)은 말뭉치를 치료 자료로 활용해 본 경험이
있었고, 음운론, 형태 및 구문론, 의미론과 관련된 영역의 치료 시 사용한 것으로 알 수 있다. 연구 자료로 활용한 응답자는 3명 (37.5\%)으로, 음운론, 의미론, 화용론과 관련된 영역의 연구를 진행하였다. 응답자의 4 명(50.0\%)은 응용된 연구 결과를 활용 하거나 말뭉치를 직접 설계, 수집 및 활용하였으며, 공개되어 있 는 세종 말뭉치 등을 직접 활용한 경우도 3명(37.5\%)이었다. 또 한 말뭉치를 활용한 교재 및 교구를 활용한 경우도 1명(12.5\%) 이었다.

말뭉치에 대한 요구도 및 사용 목적

Table 4와 같이 165 명(94.8\%)이 말뭉치를 활용할 의사가 있

Table 2. Perception and utilization experience of corpus

\begin{tabular}{llrr}
\hline Category & Type & $\mathrm{n}$ & $\%$ \\
\hline Perception & Yes & 41 & 23.6 \\
& No & 133 & 76.4 \\
Experience & Yes & 8 & 4.6 \\
& No & 166 & 95.4 \\
\hline
\end{tabular}

Table 3. Utilizing purpose and content, acquisition path of corpus

\begin{tabular}{clcc}
\hline Category & \multicolumn{1}{c}{ Type } & $\mathrm{n}$ & $\%$ \\
\hline Utilizing purpose & Therapy & 6 & 75.0 \\
& Research & 3 & 37.5 \\
Utilizing content & & \\
& Therapy & 2 & 33.3 \\
& Phonology & 4 & 66.6 \\
& Morphology and syntax & 3 & 50.0 \\
& Semantics & 0 & 0 \\
& Pragmatics & & \\
& Research & 1 & 33.3 \\
& Phonology & 0 & 0 \\
& Morphology and syntax & 1 & 33.3 \\
& Semantics & 1 & 33.3 \\
& Pragmatics & 3 & 37.5 \\
Acquisition path & Using like Sejong corpus & 4 & 50.0 \\
& Related research result & 1 & 12.5 \\
& Teaching materials & 4 & 50.0 \\
\cline { 2 - 4 } & Design, collection and utilization & & \\
& & &
\end{tabular}

Table 4. Demand and purpose for corpus

\begin{tabular}{llrr}
\hline \multicolumn{1}{c}{ Category } & \multicolumn{1}{c}{ Type } & $\mathrm{n}$ & \multicolumn{1}{c}{$\%$} \\
\hline Demand & Yes & 165 & 94.8 \\
& No & 9 & 5.2 \\
Utilizing purpose & Therapy & 157 & 95.2 \\
& Research & 50 & 30.3 \\
Utilizing content & Phonology & 61 & 36.9 \\
& Morphology and syntax & 99 & 60.0 \\
& Semantics & 79 & 47.8 \\
& Pragmatics & 98 & 59.3 \\
\hline
\end{tabular}


는 것으로 나타났으며, 이 중 157 명(95.2\%)은 치료 자료로 활용 할 것이라고 응답하였다. 활용 내용을 중복 가능하게 하여 조 사한 결과 형태 및 구문론 99명(60.0\%), 화용론 98명(59.3\%), 의미론 79명(47.8\%), 음운론 61명(36.9\%)으로 나타났다.

\section{발달장애인의 대표적 언어 자료}

발달장애인의 대표적 언어 자료를 중복 가능하게 하여 수집 한 결과, Table 5 에 나타난 바와 같이 문어에서는 122 명(70.1\%) 이 '교실 수업에서의 과제 수행 자료'라고 응답하였으며, '일기, 편지 등 작성자료' 106 명 $(60.9 \%$, '서술형 시험의 응답자료' 91명 (52.3\%), 'social networking service (SNS) 내 사용 언어' 69명 (39.7\%) 순이었다. 구어는 '가족 혹은 주변인과의 대화'가 132명 (75.9\%)으로 가장 높은 응답을 보였고, '특정 주제에 관한 대화' 111명(63.8\%), ‘전화' 96명(55.2\%), ‘발표’와 '토론’이 각각 73명 (42\%) 순이었다.

\section{발달장애인 말뭉치의 제공 자료}

발달장애인 말뭉치에 제공되는 개인정보

발달장애인 말뭉치에 제공되는 개인정보를 중복 가능하게 하여 수집한 결과, Table 6에 나타난 것과 같이 전체 응답자 중 170 명(97.7\%)에 해당하는 사람들이 언어 수준에 대한 정보가 필

Table 5. Representative language data

\begin{tabular}{clrc}
\hline \multicolumn{1}{c}{ Type } & $\mathrm{n}$ & $\%$ \\
\hline Written language & \multicolumn{1}{c}{ Diary, letter } & 106 & 60.9 \\
& Text in class & 122 & 70.1 \\
& Text in test & 91 & 52.3 \\
& Text in SNS & 69 & 39.7 \\
Spoken language & Conversation with family and & 132 & 75.9 \\
& other people & & \\
& Call & 96 & 55.2 \\
& Announcement & 73 & 42.0 \\
& Conversation about a specific & 111 & 63.8 \\
& topic & & \\
& Discussion & 73 & 42.0 \\
\hline
\end{tabular}

SNS: social networking service

Table 6. Personal information provided in developmental disability corpus

\begin{tabular}{clcc}
\hline \multicolumn{1}{c}{ Category } & \multicolumn{1}{c}{ Type } & $\mathrm{n}$ & $\%$ \\
\hline Personal information & Sex & 100 & 57.5 \\
& Age & 142 & 81.6 \\
& Occupation & 62 & 35.6 \\
& Language level & 170 & 97.7 \\
& IQ & 123 & 70.7 \\
& Degree of disabilities & 113 & 64.9 \\
\hline
\end{tabular}

요하다고 응답하였으며, 나이 142명(81.6\%), 지능 지수(IQ) 123 명 (70.7\%), 장애 등급 113명(64.9\%), 성별 100명(57.5\%), 직업 62명 $(35.6 \%)$ 순이었다.

\section{발달장애인 말뭉치에 제공되는 추가 정보}

발달장애인 말뭉치에 제공되는 추가 정보를 중복 가능하게 하여 조사한 결과, Table 7에 나타난 바와 같이 자료 종류에 관 해서는 '발화 상황'에 관한 정보가 필요하다는 의견이 150 명 (86.2\%)으로 가장 높았으며, '대화 참여자' 86 명(49.4\%), '과제 환 경' 78 명(44.8\%) 순이었다. 각각의 세부 내용으로는, '과제 환경' 에서 62명(79.4\%)이 '과제 유형'이 필요하다고 하였으며, '주제' 53명(67.9\%), '과제 종류' 46명(58.9\%) 순이었다. 발화 상황에서 는 '발화 장소 및 상황'이 125명(83.3\%)이 필요하다고 하였으며, '격식, 비격식 상황'이 81명(54.0\%)이었다. '대화 참여자'에서는 '상 하관계/친밀도'가 78명(90.6\%)으로 가장 높았으며, '나이' 57명 (66.2\%), '직업’ 48명(55.8\%), '성별' 40명(46.5\%) 순이었다.

\section{말뭉치 구축 시 추가적으로 고려할 점}

연구 대상자들에게 추가적으로 고려해야 할 점에 대해 서술 형으로 질문한 결과, 치료받는 대상자의 기분이나 반응속도, 말 의 속도, 발화 상황에 대한 경험 유무, 대외적인 경험, 가정환경, 언어치료경험 등과 같은 응답이 나왔다. 응답 중 앞의 질문과 동일한 응답이 나온 경우는 제외하였으며, 다문화가정의 아동 에 대한 조사도 필요하다는 응답이 나왔으나, 이는 국립국어원 의 한국어학습자 말뭉치에서 개발하고 있으므로 생략하였다.

Table 7. Additional information provided in developmental disability corpus

\begin{tabular}{clrc}
\hline Category & \multicolumn{1}{c}{ Type } & $\mathrm{n}$ & $\%$ \\
\hline Type of data & Task environment & 78 & 44.8 \\
& Conversation situation & 150 & 86.2 \\
& Conversation participants & 86 & 49.4 \\
Details of & Task environment & & \\
information & Type (test, homework) & 46 & 58.9 \\
& Short-answer type/descriptive type & 62 & 79.4 \\
& Topic & 53 & 67.9 \\
& Genre & 19 & 24.3 \\
& Time limitation & 30 & 38.4 \\
& Conversation situation & & \\
& Formal/informal & 81 & 54.0 \\
& Place and situation & 125 & 83.3 \\
& Conversation participants & & \\
& Sex & 40 & 46.5 \\
& Age & 57 & 66.2 \\
& Occupation & 48 & 55.8 \\
& Relation of top and bottom/intimacy & 78 & 90.6 \\
\hline
\end{tabular}




\section{DISCUSSIONS}

본 연구는 2016년 4월 한 달 동안 언어재활사 및 연구자, 관 련 전공의 대학생 174 명을 대상으로 말뭉치에 대한 인식도 및 활용 경험, 발달장애인 말뭉치 구축 시 필요한 구어 및 문어의 종류와 개인정보 등의 추가 자료에 관하여 조사한 연구이다.

본 연구를 통해서 알아본 결과는 첫째, 전체 연구 대상자의 말뭉치에 관한 인식도는 $23.6 \%$ 로 매우 낮았으며, 활용 경험이 있는 대상자는 $4.6 \%$ 에 불과하였다. 말뭉치는 언어치료학과 연 관된 사전학이나 한국어교육학 등에서도 광범위하게 쓰이며 실제 사람들에게 사용되는 언어의 모습을 보여주는 자료이지 만, 이에 관한 언어재활사 및 연구자, 관련 전공 대학생들의 지 식은 매우 부족하며 활용도 역시 낮은 모습을 보였다. 다만, 언 어 사용 빈도 등 관련 응용 연구의 많은 자료들이 언어치료학 분야에서도 쓰이고 있지만 말뭉치라는 용어의 인식도가 낮아 인지하지 못하는 경우도 많을 것이라 생각된다.

말뭉치 활용 경험이 있는 응답자 8명을 대상으로 활용 목적 및 내용, 습득 경로에 대해 조사해 본 결과, $75.0 \%$ 의 응답자는 치료 자료로 활용하였고, 형태 및 구문론과 관련된 치료 목적 으로 활용한 경우가 $66.6 \%$ 로 가장 많았으며, 의미론 $50.0 \%$, 음 운론 $33.3 \%$ 였다. 화용론과 관련된 치료 혹은 말뭉치를 활용한 교재 및 교구 활용은 $0 \%$ 로 나타났다. 연구 목적으로 활용한 경우는 $37.5 \%$ 로, 음운론, 의미론, 화용론이 $33.3 \%$ 로 나타났다. 습득 경로로는 응용 연구 결과를 활용한 것과 직접 설계, 수집 및 활용을 한 것이 $50.0 \%$ 로 가장 높았으며, 세종 말뭉치 등 공 개 말뭉치를 직접 활용한 것이 $37.5 \%$, 말뭉치를 활용한 교재 및 교구를 활용한 것이 $12.5 \%$ 로 나타났다. 이를 통해 말뭉치와 관련된 연구 및 치료는 언어의 여러 영역에서 활용되었으며, 말 뭉치를 직접 설계, 수집 및 활용한 경우도 높다는 것을 알 수 있다. 하지만 이러한 말뭉치들이 공개되지 않아 많은 연구자나 치료자들이 접근하기 어렵다는 것에 아쉬움이 있다.

발달장애인 말뭉치가 개발될 경우 활용하겠다는 의견이 전 체 응답자의 94.8\%로 나타났으며, 그중 95.2\%는 치료 자료로 활용하고 $30.3 \%$ 는 연구 자료로 활용한다는 응답이 있었다. 이 는 응답자의 $30.4 \%$ 가 언어치료 관련 연구자와 연구자 및 치료 사에 모두 포함되었는데, 이와 비슷한 비율을 보인 것은 연구자 의 대부분이 발달장애인 말뭉치가 개발되었을 때 연구에도 활 용한다는 의도를 보였음을 알 수 있다. 활용 내용으로는 형태 및 구문론이 $60.0 \%$, 화용론이 $59.3 \%$, 의미론 $47.8 \%$, 음운론 $36.9 \%$ 로, 언어의 다양한 영역에서 활용하려는 것을 알 수 있다.

둘째, 전체 응답자를 대상으로 한 발달장애인 말뭉치를 구성 할 대표적 언어 자료로 문어에서는 '교실 수업에서의 과제 수행 자료, '일기, 편지 등 작성자료, '서술형 시험의 응답자료'가 $50 \%$
이상의 높은 비율을 보였고, 구어 자료로는 '가족 혹은 주변인 과의 대화, '특정 주제에 관한 대화, '전화가 높은 비율을 보였 다. 문어 중 SNS 내 사용 언어는 상대적으로 낮은 비율을 보였 으며, 구어 중 발표 및 토론이 낮은 비율을 보였다. 응답을 살펴 보면 수업 및 치료 환경과 같은 구조화된 환경에서의 자료와 일기, 편지나 전화 등 자연스러운 상황에서 얻을 수 있는 언어 자료 등이 균형 있게 구성되어 있다는 것을 알 수 있다. 이와 같 이 구성된 경우, 말뭉치의 기본 성질인 대표성 및 균형성을 만 족시킬 수 있다는 것을 알 수 있다.

셋째, 발달장애인 말뭉치에서 개인정보 및 추가 제공 자료에 대하여 응답자들의 $97.7 \%$ 는 구어 및 문어와 같은 언어자료 외 에 대상자의 언어 수준에 대한 정보가 필요하다고 응답하였으 며, 나이, 지능 지수, 장애 등급 또한 높은 비율의 응답을 보였 다. 추가 자료의 종류로는 발화 상황에 대한 정보가 $86.2 \%$ 로 가장 높은 비율을 보였으며, 세부 내용으로는 발화 장소 및 상 황, 격식/비격식 상황에 대한 정보가 필요하다는 것이 높은 응 답률을 보였다. 과제 환경에서는 과제 유형 및 주제, 과제 종류 가 높은 응답을 보였고, 대화 참여자에서는 상하관계/친밀도, 나이, 직업이 높은 응답을 보였다. 기타 고려해야 할 점으로는 치료받는 대상자의 기분이나 반응속도, 말의 속도, 발화 상황 에 대한 경험 유무, 대외적인 경험, 가정환경, 언어치료 경험 등 과 같은 응답이 있었다. 응답자가 언어재활사 및 관련 연구자, 대학생으로 구성되어 언어 수준에 대한 정보 요구도가 높았으 며, 치료 시 고려해야 할 점과 같은 항목에 대한 응답이 많은 것으로 알 수 있었다.

다음과 같이 발달장애인 말뭉치에 대한 요구도는 매우 높으 며 여러 방면에서 응용될 것으로 기대되나 아직까지는 인식 및 활용도가 매우 낮은 것으로 결론 내릴 수 있다. 따라서 말뭉치 에 대한 언어치료 현장에서의 교육 및 여러 후속 연구들이 이 루어져야 할 것으로 제안한다. 또한 향후 발달장애인의 말뭉치 를 구축할 때, 본 연구의 결과를 토대로 발달장애인 언어가 구 현되는 여러 맥락에 걸쳐서 구축을 하는 것이 바람직할 것이라 고 제안한다.

본 연구의 제한점과 함께 후속 연구를 위한 제언을 하고자 한다. 첫째, 본 연구의 모든 응답은 자기보고 형식의 질문지로 이루어졌으며 인터넷 혹은 모바일로 이루어졌다. 말뭉치에 대 한 설명이 설문지 내에서만 이루어졌기 때문에 이해도가 부족 한 상태의 응답이 많이 보였다. 따라서 후속 연구에서는 말뭉치 에 대한 충분한 설명 및 응답자의 이해가 이루어진 후에 응답 이 이루어져야 할 것으로 보인다.

둘째, 본 연구의 응답자 중 $61.5 \%$ 가 치료 경력 1 년 미만의 치 료사였으며, 1 급 언어치료사의 비율도 $3.4 \%$ 밖에 되지 않았다. 따라서 많은 언어치료 임상 경력이 말뭉치 이해 및 사용을 높 
이는지, 연구자로서의 경험이 말뭉치 이해 및 사용을 잘 할 수 있는 것인지 알 수 없다. 따라서 후속 연구에서는 임상 경력이 많은 치료사 및 연구자나 1 급 언어재활사의 응답을 더 확보하 여 학문적으로 많은 연구를 하는 연구자와 치료를 주로 하는 치료사 간 또는 임상 경력에 따른 비교를 하는 연구도 흥미로 운 결과를 도출해 낼 수 있으리라 생각한다.

본 연구의 결과가 발달장애인의 말뭉치 구축에 대한 기초 자 료로뿐만 아니라, 말뭉치 구축이 발달장애인의 실제 생활에 도 움이 되며, 말뭉치에 대한 언어재활사의 인식을 높일 수 있는 계기가 되었기를 기대한다.

중심 단어 : 발달장애·말뭉치·언어재활사·의사소통.

\section{REFERENCES}

Choe, J. W. (2014). Corpora and linguistic research: Recent trends. Korean Linguistics, 63, 71-102.

Kang, H. W., Kim, S. J., Kim, I. H., Kim, J. S., Ahn, K. W., Lee, D. E., et al. (2015). 2015 project on basic research and construction of the Korean learner corpus. Seoul: National Institute of Korean Language.

Kim, D. G., Cho, Y. O., \& Lim, J. R. (2016). A study on the effective measures for the right to vote of socially disadvantaged. Seoul: National Election Commission.

Kim, S. H., Lee, Y. H., Hwang, J. H., Oh, M. A., Lee, M. K., Lee, N. H, et al. (2014). 2014 National Survey of the Disabled Presons. Sejong: Korea Institute for Health and Social Affairs.

Kim, W. S., Lee, S., Seo D. H., \& Eom, Y. J. (2017). A study on the research trends related to the linguistic characteristics of the people with intellectual disability in Korea: 2000 to 2016. Journal of Intellectual Disabilities, 19(1), 21-43.

Kim, Y. D. \& Cho, K. H. (2013). Big data and statistics. Journal of the Korean Data and Information Science Society, 24(5), 959-974.

Kwon, J. S. (2016). A survey and analyze on the acceptance attitudes and perception of the parents of students with disability on "The Act Support and Guarantee of Right for Persons with Developmental Disability". The Journal of the Korean Association on Developmental Disabilities, 20(3), 23-42.

Kwak, Y. J., Park, J. H., Kim, H. S., Yoo, H. J., Lee, H. J., Kwon, T. S., et al. (2015). A research on Korean learner's corpus building assistant toolkit development. Seoul: National Institute of Korean Language.

Nam, K. I. (2014). Research trends and issues on corpus-linguistics-as-theory. Korean Semantics, 46, 163-187.

Seo, S. K., Kang, H. H., Lee, K. H., Ahn, E. J., \& Hong, H. R. (2010). Designing of Korean learner corpus construction. Seoul: National Institute of Korean Language.

Seo, S. K. \& Han, Y. K. (1999). Introduction to Korean informatics. Seoul: Thaehaksa. 